\title{
Benign Cystadenofibroma Masquerading Malignant Tumor
}

\author{
Sunita Dubey ${ }^{1}$, Aayushi Kaushal ${ }^{2}$, Haradanahalli N Pavithra ${ }^{3}$
}

\begin{abstract}
Introduction: Cystadenofibroma of the ovary is a rare benign epithelial tumor of the ovary that impersonates with malignant features like solid nodule and papillae on imaging as well on gross examination of the specimen.

Case description: A 20-year-old unmarried girl visited our outpatient department with a complaint of pain in the lower abdomen for the last 1 year. Transabdominal ultrasonography revealed a well-defined complex cyst of about $9.4 \times 7.4 \mathrm{~cm}$ size with a small echogenic nodule of 1.74 $\times 0.8 \mathrm{~cm}$ involving the left ovary. On color Doppler, there was an absence of increased internal vascularity. The right ovary was normal. CA 125 was within normal limits. She underwent laparoscopy, multiple solid papillae were seen after removal of a cyst, left salpingo-oophorectomy was done due to suspicion of malignancy. However, the final histopathology report revealed benign cystadenofibroma of the ovary.

Conclusion: Although there are no confirmatory imaging diagnostic tests to differentiate benign cystadenofibroma from malignant pathology, however, absent blood flow is significantly associated with the benign nature of ovarian cystadenofibromas despite solid nodule and papillae within it. An intraoperative frozen section may help in diagnosis and prevent extensive surgery in patients.

Keywords: Adnexal mass, Benign epithelial tumor of ovary, Cystadenofibroma of ovary, Frozen section, Magnetic resonance imaging of adnexal mass.

The Journal of Medical Sciences (2020): 10.5005/jp-journals-10045-00147
\end{abstract}

\section{INTRODUCTION}

Ovarian cystadenofibromas are a rare type of benign surface epithelial tumor of the ovary. They have been reported in each phase of life with a wide age distribution of $19-75$ years. ${ }^{1,2}$ Various histopathological patterns like a mucinous, serous, clear cell, mixed, and endometroid type have been mentioned in the literature. ${ }^{3}$ Exact cause of benign cystadenofibroma of the ovary are unknown and often considered to occur spontaneously. It has been incidentally found as an asymptomatic pelvic mass during routine gyne examination, whereas chronic pain abdomen and bleeding per vaginum were the commonest presenting symptoms reported in the literature. ${ }^{1}$

CA 125 was usually found within normal limits in all cases but raised serum estradiol levels have been reported in an endometroid type of cystadenofibroma of the ovary. ${ }^{4}$ On imaging studies, these benign tumors can be predominantly cystic, complex cystic with variable amounts of solid components or predominantly solid. Preliminary imaging including ultrasonography (USG) or computed tomography (CT) scan may not prove valuable in differentiating them from malignant tumors. The present case is brought for discussion to know different imaging findings of cystadenofibroma and use of intraoperative frozen section to know the nature of the adnexal mass, particularly in a young patient.

\section{Case Description}

A 20-year-old unmarried girl visited our outpatient department with a complaint of pain in the lower abdomen for the last 1 year. The patient was clinically stable. On examination, her pulse was 82 /minutes and her blood pressure was $110 / 80 \mathrm{~mm} \mathrm{Hg}$. Her respiratory and cardiovascular systems were normal. On abdominal examination, approximately $16-18$ weeks size cystic, a non-tender mass was felt with restricted mobility. Her routine investigations were within normal limits. Tumor markers, CA 125 was $11.5 \mu / \mathrm{mL}$, alpha-fetoprotein was $0.515 \mathrm{IU} / \mathrm{mL}$, and carcinoembryonic antigen (CEA) was $0.69 \mathrm{lU} / \mathrm{mL}$.

\footnotetext{
1,2Department of Obstetrics and Gyanecology, Government Medical College and Hospital, Chandigarh, India

${ }^{3}$ Department of Pathology, Government Medical College and Hospital, Chandigarh, India
}

Corresponding Author: Aayushi Kaushal, Department of Obstetrics and Gyanecology, Government Medical College and Hospital, Chandigarh, India, Phone: +91 8146887529, e-mail: kaushalaayushi@ gmail.com

How to cite this article: Dubey S, Kaushal A, Pavithra HN. Benign Cystadenofibroma Masquerading Malignant Tumor. J Med Sci 2020;6(1):19-21.

Source of support: Nil

Conflict of interest: None

Transabdominal sonographic findings revealed a well-defined complex cystic lesion of size $9.4 \times 7.4 \mathrm{~cm}$ arising from the left ovary with a small echogenic nodule of $1.74 \times 0.8 \mathrm{~cm}$ was present eccentrically. Residual left ovarian tissue was seen along its posterior and superolateral margin. On color Doppler, there was no internal vascularity seen in it (Fig. 1). The decision for laparoscopic cystectomy was taken, with prior consents for laparotomy and left salpingo-oophorectomy, if needed. Intraoperatively, the upper abdomen was normal. A left ovarian cyst with a smooth outer surface was seen. Uterus and right ovary were normal. There was no evidence of ascites. Peritoneal fluid was sent for cytology. Laparoscopic cystectomy was done. On gross examination, the outer surface of the cyst was smooth. The cyst was filled with clear fluid. On the cut section, the inner surface of the cyst showed the presence of multiple solid papillary growths. In view of suspicion of malignancy, she underwent left-sided salpingo-oophorectomy (Fig. 2).

Detailed histopathological examination reported the tissue to be a benign papillary serous cystadenofibroma, negative for malignancy (Fig. 2). Peritoneal cytology was reported as negative 
for malignant cells. Her postoperative period was uneventful and she was discharged on day 2 of surgery. She has been asymptomatic since 1 year of follow-up after surgery.

\section{Discussion}

Benign cystadenomas are the most frequent surface epithelial tumor of the ovaries, whereas ovarian cystadenofibromas account

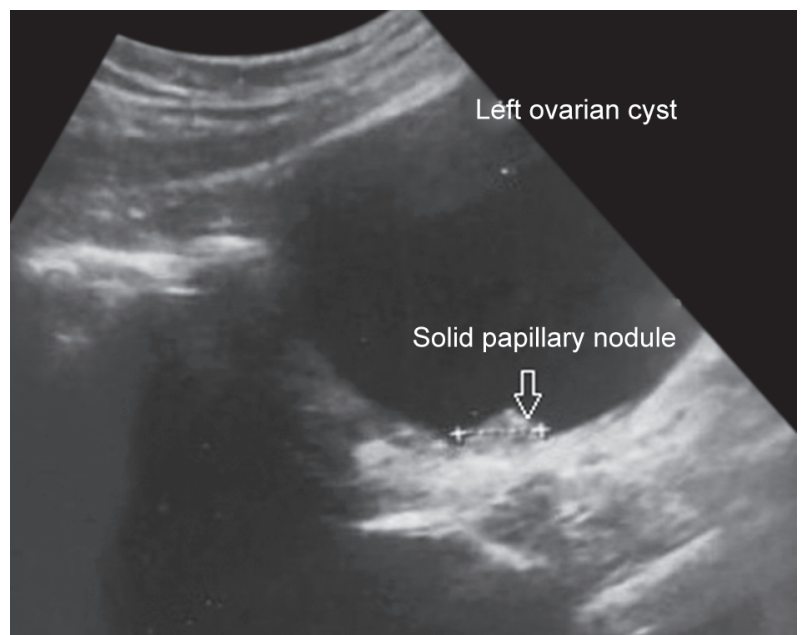

Fig. 1: Ultrasonography revealed left ovarian cyst with one solid papillary nodule at the periphery for approximately $1.7 \%$ of all benign tumors. ${ }^{5}$ There is no specific age preference for this tumor, a patient may be asymptomatic mass or may present with pain abdomen and irregular bleeding per vaginum. Involvements of the bilateral ovary have been reported either with similar or different pathology. ${ }^{6}$

Histopathologically these tumors show some evidence of fibrous tissue in the stroma, the suffix fibroma is attached as in serous adenofibroma when a fibrous portion is larger than the cystic component, whereas cystadenofibroma is called when the tumor also contain cyst of more than 1 of more than $2 \mathrm{~cm}$ in size. ${ }^{3}$ These tumors are classified as serous, endometrioid, mucinous, clear cell, and mixed categories according to the type of epithelial cell they contain, whereas the degree of epithelial proliferation and its relation to the stromal component classified them into benign, borderline, or malignant tumors.,

Ovarian cystadenofibromas are significantly variable from cystic, uni- or multilocular with or without solid component either in the form of papillae or mural nodule thus, mimic malignant neoplasms on imaging as well on intraoperative findings. Imaging in the present case revealed one unilocular cyst with a single solid papilla which was appeared to be benign by simple rule and ADNEX model while intraoperative findings revealed multiple solid papillae. The discrepancy in imaging and intraoperative finding in the present case could be due to the use of transabdominal ultrasound rather than transvaginal ultrasonography or magnetic resonance imaging (MRI). Due to these complex and misleading
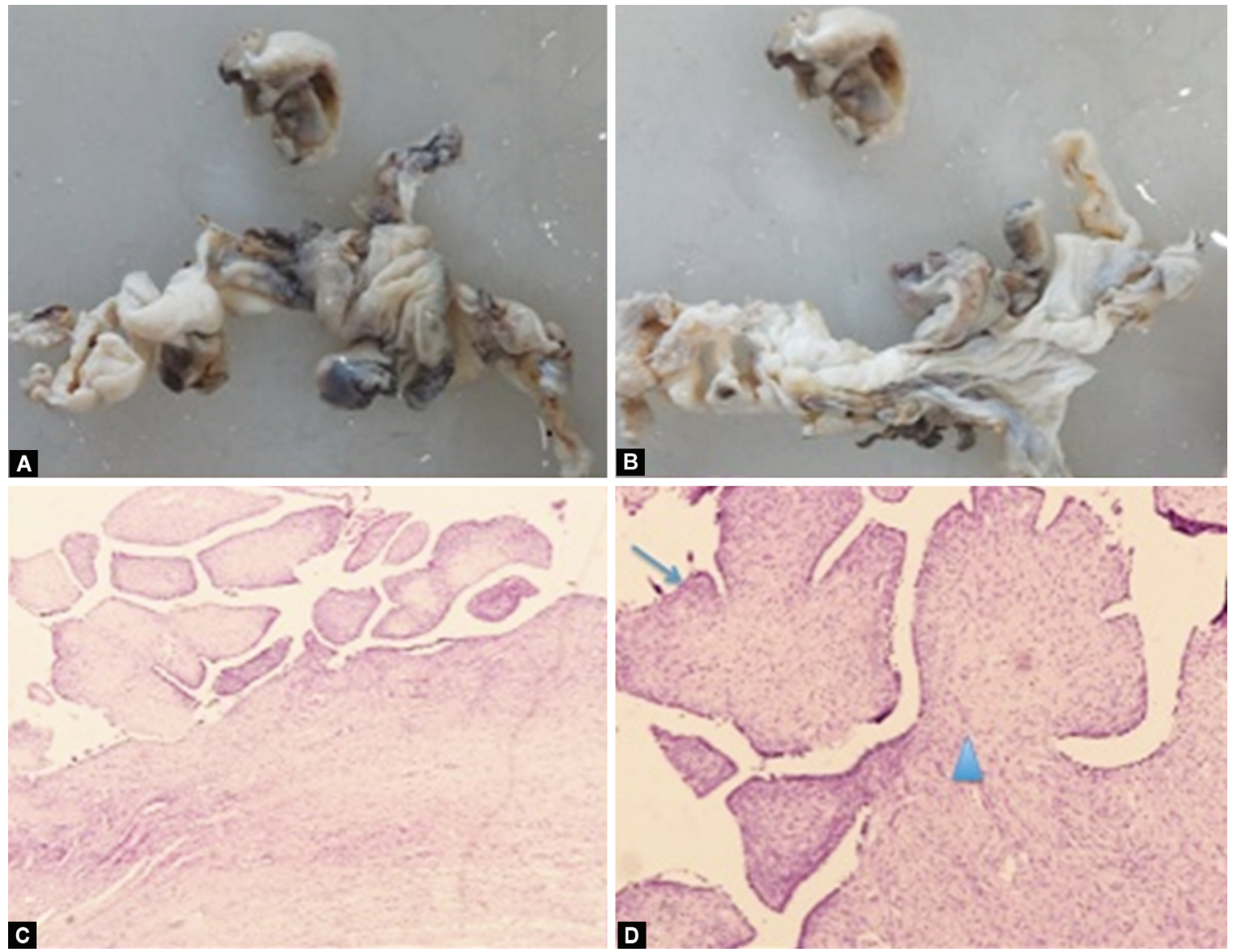

Figs 2A to D: Gross and HPE of cystadenofibroma: (A and B) Gross photo from left ovary shows an already cut open cyst with focal papillary excrescences; ( $C$ and D) 40x and 100x magnified ( $\mathrm{H}$ and $\mathrm{E}$ stain) images of papillae lined by bland serous lining epithelium (arrow) and prominent fibrous stroma (arrowhead) 
features, extensive surgeries have been done and reported in young patients. ${ }^{9-11}$

Therefore, authors' have retrospectively correlated various imaging pictures with the histopathological finding in confirmed cases of benign cystadenofibroma to conclude. Outwater et al. ${ }^{12}$ have first reported the unique description of the fibrous component of ovarian fibromas and cystadenofibromas on T2-weighted images of MRI where the fibrous component revealed very low signal intensity similar to skeletal muscle. Goldstein et al. ${ }^{13}$ retrospectively reviewed the transvaginal ultrasonography report of 32 cases where he did not find any typical appearance on transvaginal ultrasonography and color Doppler imaging. However, the presence of unilocular cyst with one or more solid avascular projection was found $100 \%$ reliable in diagnosing $69 \%$ of cases of benign ovarian cystadenofibromas. The presence of blood flow, papilla, or nodule in ovarian cyst usually raise the suspicion of malignancy; however, solid areas in ovarian cystadenofibromas appears avascular due to hard, fibrous tissue, and presence of which appears hyperechoic due to reflection of a sound wave and gives acoustic shadow distal to them on ultrasound imaging. In their series, $31 \%$ of benign cases revealed a variable amount of blood flow in a multilocular heterogeneous cyst, hence could not be sure of benign pathology. Another large retrospective review of ultrasound findings in confirmed cases of cystadenofibroma revealed avascular unilocular solid cyst with one or more papilla as the most common ultrasound finding followed by acoustic shadowing along with reduced blood flow. ${ }^{14}$

Byun et al. ${ }^{5}$ also reported borderline or malignant features in 14 out of 32 confirmed cases of benign ovarian cystadenofibroma (serous in 13 and endometrioid in 1) on CT or MRl; hence, incorrectly misdiagnosed as a malignant mass on imaging studies. Contrast enhancement in the solid area was reported greater than skeletal muscle by Byun et al., ${ }^{5}$ whereas in a case series by Tang et al. ${ }^{6}$ majority of solid areas revealed low T2 signal intensity and minimal contrast enhancement less than that of the myometrium. Jung et al. ${ }^{15}$ reported diffuse and partial thickening of cyst wall in predominantly cystic mass as dark signal intensity on T2-weighted $\mathrm{MRI}$ and the presence of solid fibrous component displayed characteristic of a black sponge-like image that was also described by Takeuchi et al. ${ }^{16}$

On MRI whenever complex ovarian or adnexal lesions are seen with a black sponge-like image with low signal intensity on T2-weighted sequences along with normal tumor markers, the probability of benign tumor should be kept in mind and frozen sections may direct appropriate surgical management by establishing a precise intraoperative diagnosis of ovarian masses that might prevent a young patient from unnecessary oophorectomy like in the present case where cystectomy could have been sufficed as residual normal left ovarian tissue was seen along its posterior and superolateral margin.

\section{ConcLusion}

Although ovarian cystadenofibroma have variable and complex imaging picture, however, normal tumor markers with absent or no extra blood flow in an otherwise malignant looking adnexal mass significantly point toward the benign nature of the cyst.

Resource of an intraoperative frozen section should be utilized to avoid extensive surgery in young patients, especially when there are discrepancies in imaging and intraoperative findings.

\section{References}

1. Groutz A, Wolman I, Wolf Y, et al. Cystadenofibroma of the ovary in young women. Eur J Obstet Gynecol Reprod Biol 1994;54(2):137-139. DOI: 10.1016/0028-2243(94)90253-4.

2. Fatum M, Rojansky N, Shushan A. Papillary serous cystadenofibroma of the ovary -is it really so rare? Int J Gynaecol Obstet 2001;75(1):8586. DOI: 10.1016/S0020-7292(01)00415-5.

3. Czernobilsky B, Borenstein R, Lancet M. Cystadenofibroma of the ovary. A clinicopathologic study of 34 cases and comparison with serous cystadenoma. Cancer 1974;34(6):19711981. DOI: 10.1002/1097-0142(197412)34:6<1971::AIDCNCR2820340616>3.0.CO;2-N.

4. Singh N, Tripathi R, Mala YM, et al. Large functional benign endometroid cystadenofibroma of the ovary leading to endometrial cystic glandular hyperplasia and postmenopausal bleeding. BMJ Case Rep 2013;2013, bcr2013010323 10.1136/bcr-2013-010323.

5. Byun JY, RhaSE, Jung SE, et al.CT and MRI findings of cystadenofibromas of the ovary. Eur Radiol 2004;14(5):798-804. DOI: 10.1007/s00330-0032060-z.

6. Tang YZ, Liyanage S, Narayanan P, et al. The MRI features of histologically proven ovarian cystadenofibromas-an assessment of the morphological and enhancement patterns. Eur Radiol 2013;23(1):48-56. DOI: 10.1007/s00330-012-2568-1.

7. Scully RE, Young RH, Clement PB. Atlas of Tumor Pathology. 3rd Series, Washington, DC, USA: Armed Forces Institute of Pathology; 1998.

8. Compton HL, Finck FM. Serous adenofibroma and cystadenofibroma of the ovary. Obstet Gynecol 1970;36(4):636-645.

9. Lee DH. A case of mucinous cystadenofibroma of the ovary. Case Rep Obstet Gynecol 2014;2014:130530. DOI: 10.1155/2014/130530.

10. Shukla $S$, Srivastava $D$, Acharya $S$, et al. Serous adenofibroma of ovary: an eccentric presentation. J Can Res Ther 2015;11(4):1030. DOI: 10.4103/0973-1482.150419.

11. Singh NV, Venkatraman I, Roshni J, et al. Benign serous cyst adenofibroma of ovary - case report. IOSR J Dent Med Sci 2017;16(4):01-03. DOI: 10.9790/0853-1604010103.

12. Outwater EK, Siegelman ES, Talerman A, et al. Ovarian fibromas and cystadenofibromas: MRI features of the fibrous component. J Magn Reson Imaging 1997;7(3):465-471. DOI: 10.1002/jmri.1880070303.

13. Goldstein SR,Timor-TritschIE, Monteagudo A, etal.Cystadenofibromas: can transvaginal ultrasound appearance reduce some surgical interventions? J Clin Ultrasound 2015;43(6):393-396. DOI: 10.1002/ jcu.22241.

14. Virgilio BA, De Blasis I, Sladkevicius P, et al. Imaging of gynecological disease(16): clinical and ultrasound characteristics of serous cystadenofibromas in the adnexa. Ultrasound Obstet Gynecol 54(6):823-830. DOI: 10.1002/uog.20277.

15. Jung DC, Kim SH, Kim SH. MR imaging findings of ovarian cystadenofibroma and cystadenocarcinofibroma: clues for differential diagnosis. Korean J Radiol 2006;7(3):199-204. DOI: 10.3348/kjr.2006.7.3.199.

16. Takeuchi M, Matsuzaki K, Kusaka M, et al. Ovarian cystadenofibromas: characteristic magnetic resonance findings with pathologic correlation. J Comput Assist Tomogr 2003;27(6):871-873. DOI: 10.1097/00004728-200311000-00007. 